\title{
Offene Leistenhernienreparation
}

\section{K. Junge ${ }^{1}$, J. Otto ${ }^{1}$, J. Conze ${ }^{2}$}

${ }^{1}$ Klinik für Allgemein-, Viszeral- und Minimalinvasive Chirurgie, Medizinisches Zentrum StädteRegion Aachen GmbH

2 Hernienzentrum München

Die Leistenhernie stellt mit einem Anteil von 10-15\% aller chirurgischen Eingriffe und ca. 20 Millionen Eingriffen weltweit pro Jahr eine der häufigsten chirurgischen Interventionen dar. So werden in Deutschland derzeit jährlich ca. 275000 Leistenhernien operiert. Aufbauend auf die dezidierte Darstellung der minimalinvasiven Operationsverfahren
(Köckerling et al. 2012) werden in diesem Beitrag die gebräuchlichsten offenen Verfahren zur Therapie der Leistenhernie (Lichtenstein, Shouldice) detailliert abgehandelt, wenngleich nach den aktuellen Leitlinien (Simons et al. 2009; Miserez et al. 2014) bei den meisten Patienten eine Netzimplantation empfohlen wird.

\section{Ätiologie, Pathophysiologie}

25\% aller Männer und 2\% aller Frauen müssen während ihres Lebens mit dem Auftreten einer Leistenhernie rechnen. Neben der Manifestation im Kindes- und Jugendalter liegt der Gipfel im höheren Erwachsenenalter (> 40 Jahre). Bilaterale Befunde sind in $15-30 \%$ zu erwarten. Pathophysiologisch zeigt sich ein erhöhtes Risiko bei Rauchern, Patienten mit einer positiven Familienanamnese, persistierend offenem Processus vaginalis, Kollagenerkrankungen, Patienten mit einem abdominellen Aortenaneurysma, nach Appendektomie, nach Prostatektomie, Patienten mit Aszites, Peritonealdialyse oder chronisch obstruktiver Lungenerkrankung (Simons et al. 2009).

\section{Anatomie}

Der Leistenkanal dient als Durchtrittsstelle des Funiculus spermaticus beim Mann bzw. des Lig. teres uteri bei der Frau. Darüber hinaus ziehen der N. ilioinguinalis und der R. genitalis des N. genitofemoralis durch den Leistenkanal. Der Leistenkanal erstreckt sich vom Anulus inguinalis profundus (innerer Leistenring) bis zum Anulus inguinalis superficialis (äußerer Leistenring). Die kraniale Begrenzung des Kanals bilden die Unterkanten des
M. obliquus internus und des M. transversus abdominis sowie der Unterrand der Rektusscheide. Die Vorderwand des Leistenkanals wird durch die Aponeurose des M. obliquus externus gebildet. Nach dorsal wird der Kanal von der Fascia transversalis und dem Peritoneum begrenzt. Das Lig. inguinale bildet schließlich den Boden des Leistenkanals.

- Zu den gerade für die offenen Verfahren relevanten nervalen Strukturen gehören der N. ilioinguinalis, der R. genitalis des N. genitofemoralis und der N. iliohypogastricus (Abb. 1).

Die Bruchpforten der Leistenhernie finden sich entweder medial im Bereich der Fossa inguinalis medialis (= direkter Bruch) oder lateral in der Fossa inguinalis lateralis (= indirekter Bruch), wobei auch kombinierte Befunde vorkommen. Eine Sonderform stellt die Schenkel- oder Femoralhernie dar, deren Bruchpforte sich unterhalb des Lig. inguinale medial der Gefäße in der Lacuna vasorum befindet (Abb. 2). 


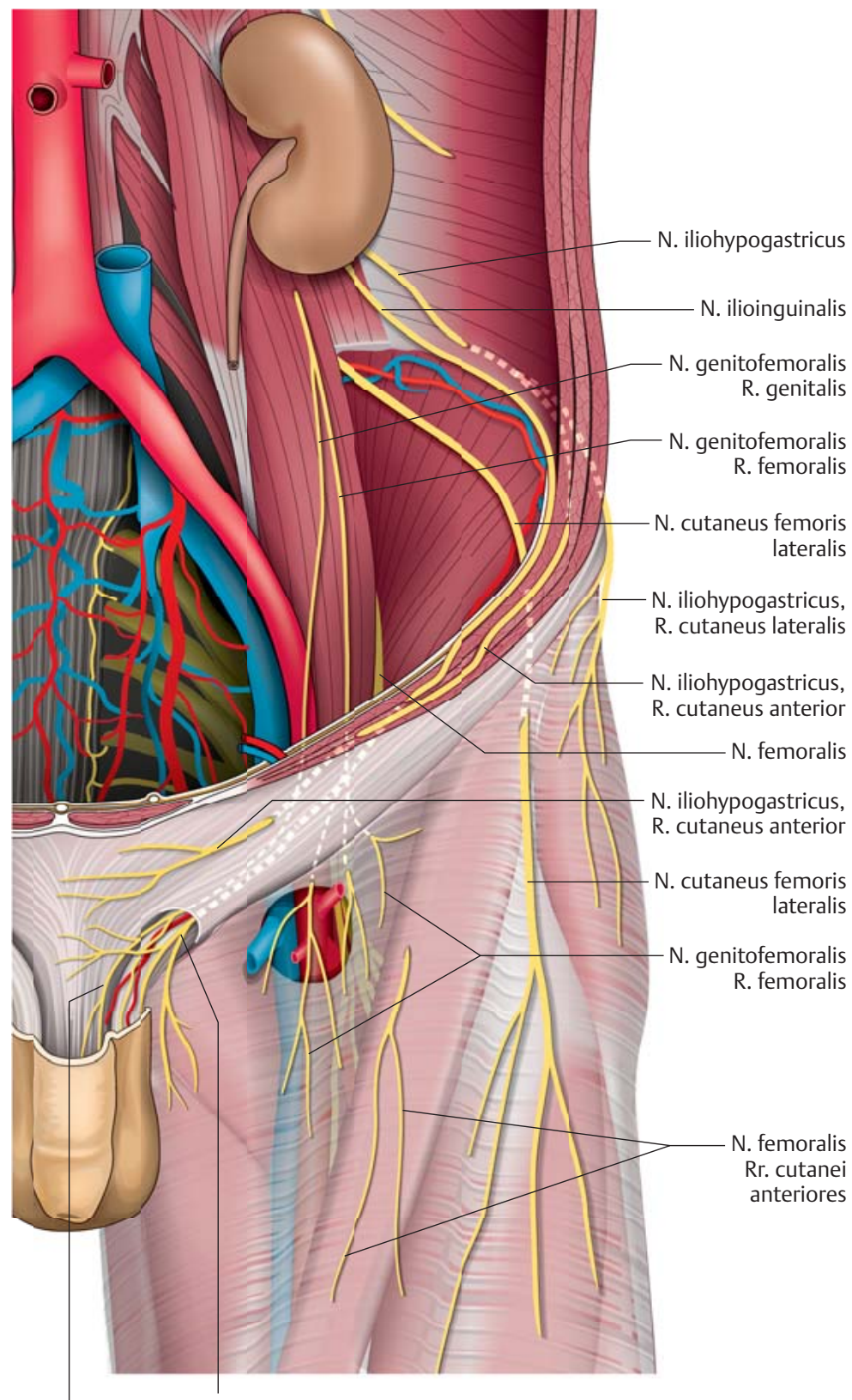

N. genitofemoralis N. ilioinguinalis R. genitalis

Abb. 1 - Sensible Innervation der Leistenregion (aus: Köckerling F, Jacobs D, Grund S et al. Prinzipien der minimalinvasiven Chirurgie bei Hernien Teil 2. Allgemein- und Viszeralchirurgie up2date 2012; 2: 99-115).

\section{Klinik}

Die oftmals asymptomatische unkomplizierte Leistenhernie kann als Zufallsbefund im Rahmen einer klinischen oder sonografischen Untersuchung auffallen. Symptomatisch wird eine Leistenhernie häufig lediglich als diskrete, primär gut reponible Schwellung oder mit unspezifischen ziehenden Leistenschmerzen unter Belastung
(Bauchpresse, Lasten heben usw.) vom Patienten wahrgenommen. In diesem Zusammenhang können folgende Komplikationen auftreten: Bei einer Irreponibilität zeigt sich die Schwellung persistierend, bei einer Inkarzeration finden sich zusätzlich starke Schmerzen mit einer tastbaren, prall elastischen Schwellung in der Leistenregion. Es droht eine Ileussymptomatik bis hin zur Darmperforation und Peritonitis.

Bei einer akuten Inkarzeration kann eine manuelle Reposition der Bruchgeschwulst innerhalb der ersten Stunden nach Inkarzeration versucht werden. Um eine manuelle Verletzung des Bruchinhalts zu vermeiden, sollte dies unter sonografischer Kontrolle der anatomischen Verhältnisse mit Lokalisation der Bruchlücke durchgeführt werden. Nach gelungener Reposition sollte eine stationäre Überwachung und möglichst eine zeitnahe operative Hernienreparation im selben Aufenthalt erfolgen. Bei erfolglosem Repositionsversuch muss eine operative Behandlung notfallmäßig erfolgen.

\section{Diagnostisches Vorgehen}

\section{Die klinische Untersuchung am stehenden Patienten} unter Bauchpresse (Valsalva-Manöver) ermöglicht die Beurteilung einer Bruchpforte und das Palpieren eines Bruchs. Hier kann gleichzeitig die Frage der Reponibilität geklärt werden. In Kombination mit der Sonografie erlaubt sich somit eine sichere Aussage über das Vorliegen eines Bruchs, die Größe der Bruchpforte, den Bruchinhalt, die Reponibilität und das Vorliegen einer Inkarzeration. Nur in Ausnahmefällen, z. B. bei Voroperationen in der Leistenregion oder ausgeprägter Adipositas mit entsprechender Fettschürze, macht eine CT- oder MRT-Untersuchung Sinn.

Klassifikation. Eine einheitliche Klassifikation der Leistenhernie ist erforderlich, um die Reproduzierbarkeit und Vergleichbarkeit klinischer Studien zu gewährleisten. Grundlage ist die Erkenntnis, dass die einzelnen Bruchformen ein durchaus unterschiedliches Risikoprofil für das Entstehen eines Rezidivbruchs aufweisen. Nur eine eindeutige Einteilung der differenten Bruchformen ermöglicht die kritische Analyse der Ergebnisse. Hierzu wurde 2007 die Klassifikation der European Hernia Society (EHS) eingeführt (Tab. 1). Dabei wird neben der Lokalisation (medial, lateral, femoral) die Größe der Bruchpforte mittels Fingerspitzenkuppe eingeteilt, angelehnt an die Aachener Klassifikation:

- $1=<1$ Fingerspitzenkuppe $(\leq 1,5 \mathrm{~cm})$

- $2=1-2$ Fingerspitzenkuppen $(1,5-3,0 \mathrm{~cm})$

- mehr als 2 Fingerspitzenkuppen $(>3 \mathrm{~cm})$ 


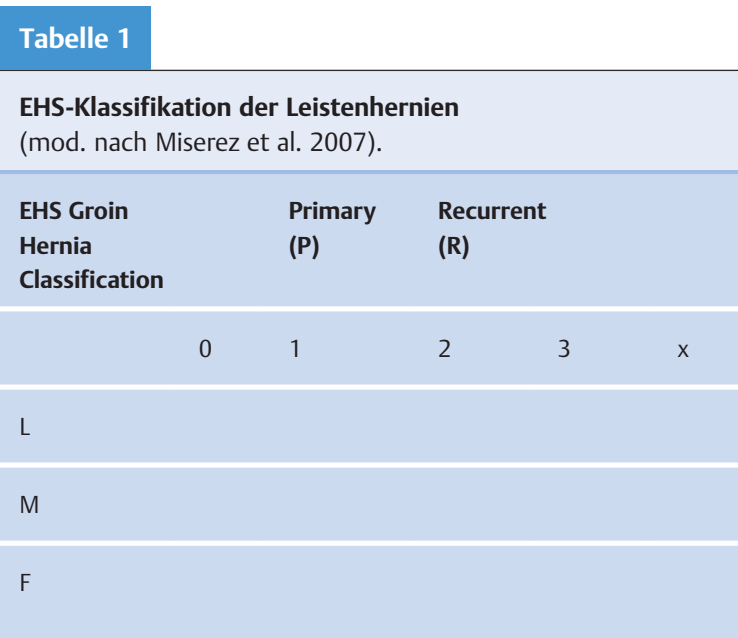

Mittels P und R wird das Vorliegen einer Primär- bzw. einer Rezidivhernie angegeben. Kombinierte Brüche sollen mit den beiden Komponenten separat abgebildet werden. So ist eine PL3M1-Hernie eine kombinierte Primärhernie mit einem kleinen direkten und einer großen indirekten Komponente.

\section{Indikationsstellung und Verfahrenswahl}

Das chirurgische Dogma „die Diagnose einer Leistenhernie bedingt die Indikation zur Reparation“ ist heutzutage nicht mehr haltbar. So konnten große prospektiv randomisierte Studien zeigen, dass ein Watchful-Waiting-Konzept bei jüngeren Patienten mit asymptomatischen oder minimal symptomatischen Hernien praktizierbar und auch vertretbar ist (Fitzgibbons et al. 2006). Im Rahmen dieser Studie an 720 männlichen Leistenbruchpatienten konnte gezeigt werden, dass es in den beiden Vergleichskollektiven keine Unterschiede im Hinblick auf den physischen Aktivitätslevel gibt und dass keine Unterschiede im Hinblick auf Komplikationen existieren. Eine weitere Studie zur Praktizierbarkeit eines Watchful-WaitingKonzepts fand jedoch auch, dass die primäre Reparation keinen signifikanten Einfluss auf die Rate chronischer Schmerzen bei asymptomatischen Patienten im Verlauf hatte (O'Dwyer et al. 2006). Weiter zeigen die Langzeitergebnisse beider Studien, dass im Follow-up ein Crossover zur Operation bei $68 \%$ bzw. $72 \%$ zumeist aufgrund einer Symptomzunahme im Verlauf zu verzeichnen war (Fitzgibbons et al. 2013, Chung et al. 2011).

\section{Die Leitlinien der Europäischen Herniengesellschaft} wurden aufgrund der Oxford-Kriterien für evidenzbasierte Wissenschaft erstellt. Demnach sind es vor allem

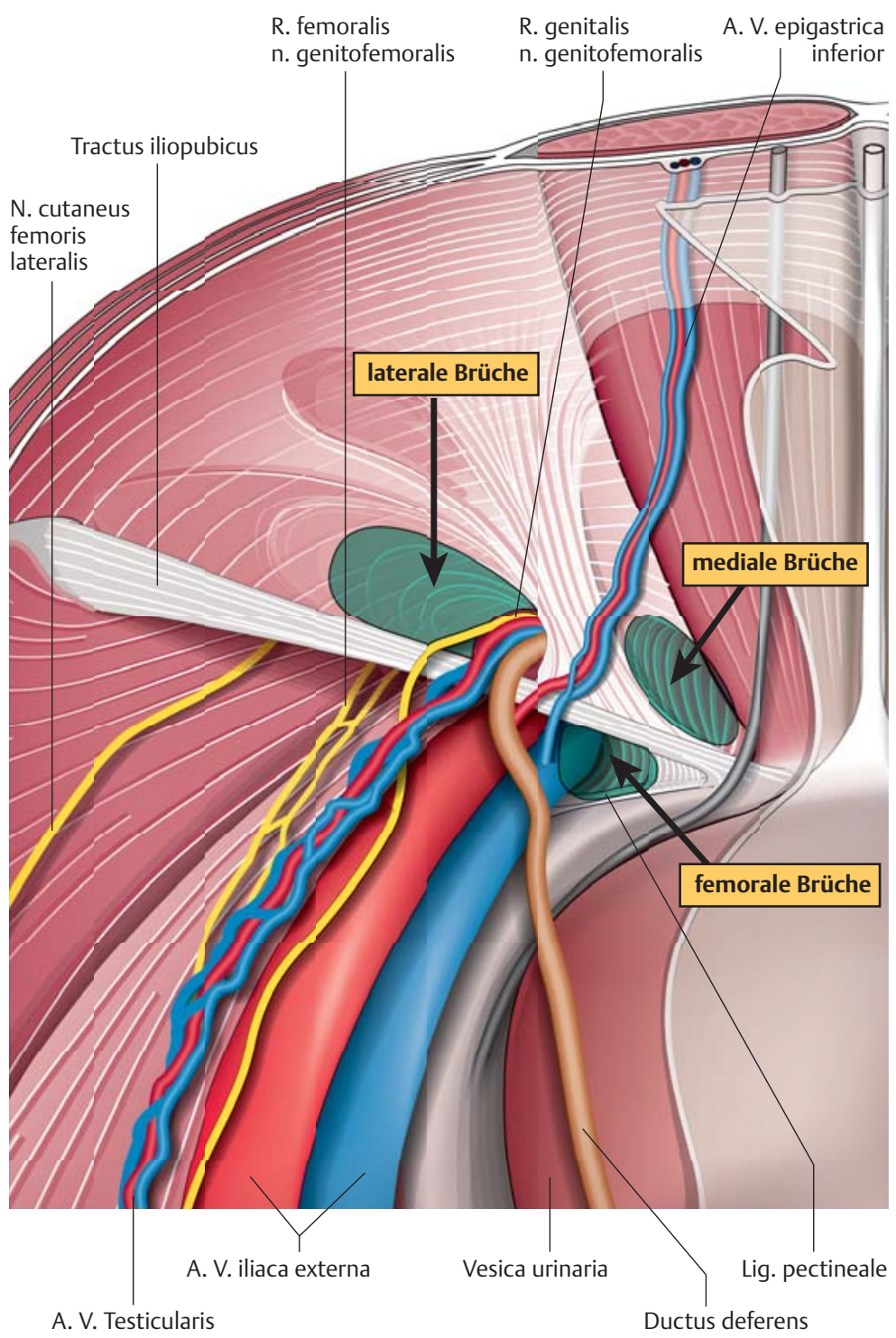

Abb. 2 - Lokalisation der Bruchpforten in der Ansicht von dorsal (aus: Köckerling F, Jacobs D, Grund S et al. Prinzipien der minimalinvasiven Chirurgie bei Hernien Teil 2. Allgemein- und Viszeralchirurgie up2date 2012; 2: 99 -115).

prospektiv randomisierte Studien und Metaanalysen, welche in die Therapieempfehlungen Eingang gefunden haben. Die Abb. 3 gibt den aktuellen Flowchart zur Versorgung einer Leistenhernie nach den Leitlinien der Europäischen Herniengesellschaft wieder (Miserez et al. 2014).

In einer retrospektiven Ergebnisstudie nach ShouldiceReparation konnten die Risikofaktoren für eine Rezidivhernie nach Nahtverfahren aufgezeigt werden (Junge et al. 2006). Demnach sind Nahtverfahren bei großen medialen primären Leistenhernien und bei Rezidivhernien überfordert und mit einer hohen Rezidivrate behaftet. Hingegen konnten für laterale und auch kleinere 


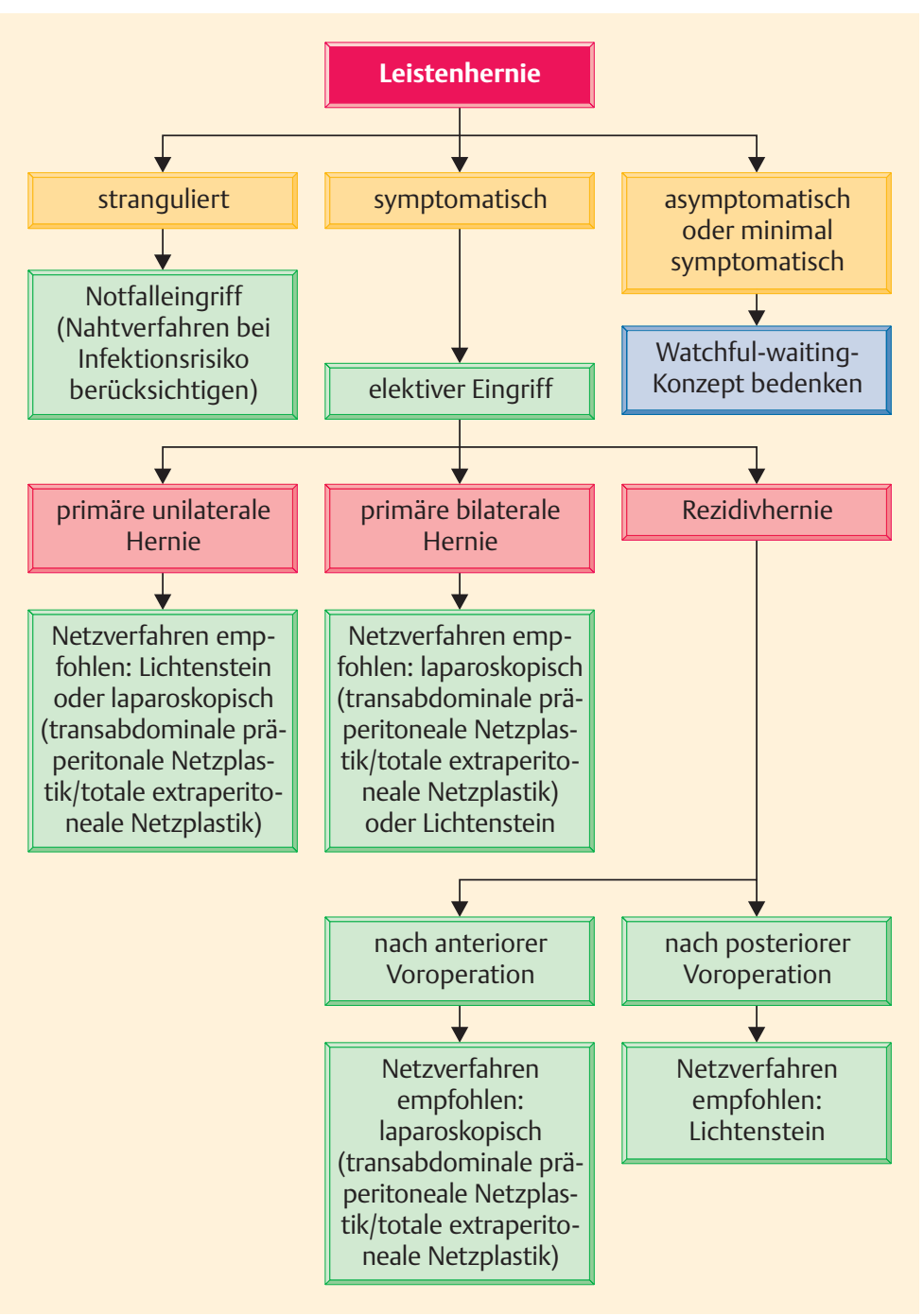

Abb. 3 - Indikationsstellung und Verfahrenswahl (mod. nach Simons et al. 2009 u. Miserez et al. 2014).

\section{Operative Therapie}

\section{Operationsvorbereitung}

Die operative Intervention ist in der Regel elektiv planbar durchzuführen. Dazu gehört die frühzeitige Aufklärung des Patienten über operative, aber auch konservative (Watchful-Waiting-Konzept) Behandlungsansätze. Im Aufklärungsgespräch müssen die typischen (auch seltenen) Komplikationen angesprochen werden.

Ein Vorteil der offenen Verfahren zur Leistenhernienreparation besteht in der Möglichkeit der operativen Intervention unter Lokalanästhesie, die bei laparoskopischen Eingriffen nicht möglich ist. Anderweitig können natürlich auch eine Intubations- oder Maskennarkose wie auch eine Spinalanästhesie durchgeführt werden. Nach aktuellen Daten und dem Update der Leitlinien der Europäischen Herniengesellschaft kann selbst bei ausgewählten älteren und ASA(American Society of Anaesthesiologists)III-Patienten die Lokalanästhesie Anwendung finden. Generell sollte bzw. muss bei jedem Patienten - auch aus abrechnungstechnischen Gründen - eine ambulante operative Intervention überdacht werden. Eine Antibiotikaprophylaxe wird für die offenen Verfahren auch unter Netzimplantation lediglich bei einer generell hohen Wundinfektionsrate (>5\%) und Risikopatienten empfohlen (Miserez et al. 2014).

\section{Lagerung}

Die Lagerung des Patienten erfolgt in Rückenlage. Durch die Platzierung eines Gelkissens unter das Becken kann die dadurch überstreckte Leiste besser exponiert werden.

\section{Lokalanästhesie}

Shouldice-Verfahren mit einem Follow-up von 10 Jahren belegt werden. Daraus hat sich das Tailoring entwickelt, die intraoperative Verfahrenswahl aufgrund der vorliegenden anatomischen Verhältnisse, der Morphologie der Hernie und dem Risikoprofil des jeweiligen Patienten. Die Überlegenheit der Shouldice-Reparation gegenüber anderen Nahtverfahren konnte dabei durch eine aktuelle Cochrane-Analyse dargestellt werden (Amato et al. 2012).

Auch wenn der Anteil an Nahtverfahren in der Hernienversorgung in Deutschland in den letzten Jahren deutlich gesunken ist, sollte jeder Chirurg, der Hernienchirurgie durchführt, dieses Verfahren beherrschen und gezielt einsetzen können.
Die Lokalanästhesie wird als Kombination aus einer Leitungs- und einer Infiltrationsanästhesie durchgeführt. Die Leitungsanästhesie zur Blockade des N. iliohypogastricus und des N. ilioinguinalis erfolgt leicht kraniomedial der Spina iliaca anterior superior. Hier können auf Faszienniveau beide Nerven suffizient blockiert werden (Abb. 4a). Additiv erfolgt eine Infiltrationsanästhesie im Bereich der geplanten Hautinzision (Abb. 4b). Intraoperativ erfolgt zusätzlich eine Blockade des R. genitalis des N. genitofemoralis am inneren Leistenring bzw. entlang der Blue Line. Zur Anwendung können gebräuchliche Lokalanästhetika unter Beachtung der Höchstmengen kommen (z. B. Naropin $7,5 \%$ ). 


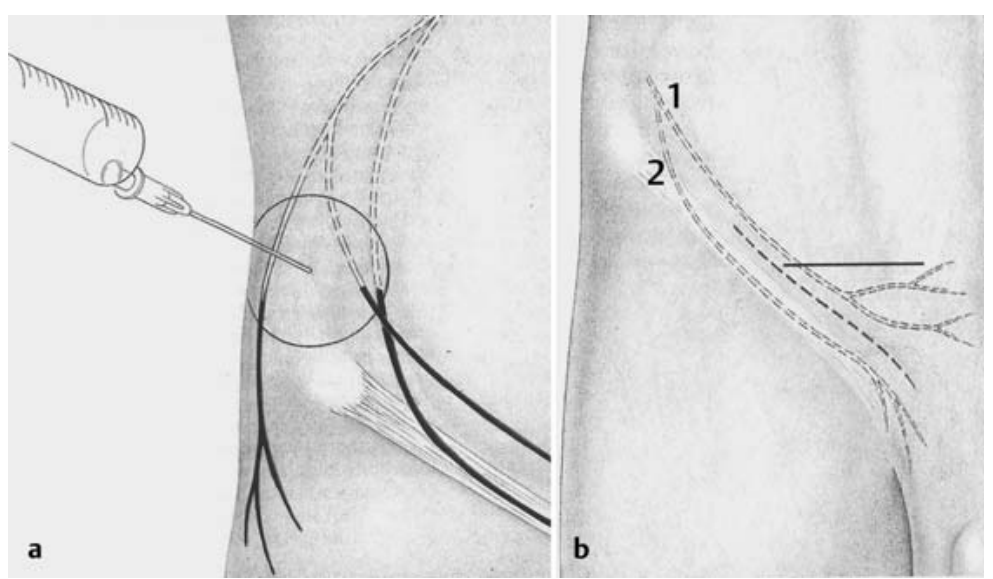

Abb. 4 - Lokalanästhesie (aus: Schumpelick M. Hernien. 4. Aufl. Stuttgart: Thieme; 2000). a Punktionsort zur Leitungsanästhesie des N. iliohypogastricus und des N. ilioinguinalis. b Infiltrationsanästhesie entsprechend der Lage der Hautinzision beim Leistenschrägschnitt oder Querschnitt in der Leistenbeugefalte. 1: N. iliohypogastricus; 2: N. ilioinguinalis.

\section{OP-Schritte und Tricks}

\section{- Präparation}

Siehe hierzu Abb. 5

Die Hautinzision kann als Leistenschrägschnitt oder Querschnitt im Bereich oberhalb der Leistenbeugefalte erfolgen, ca. ein Querfinger über der Symphyse (Abb. 5 a). In der Subkutis können regelhaft die kreuzenden Vasa epigastrica superficialia dargestellt und zwischen Klemmchen durchtrennt und ligiert werden (Abb. 5b).

Nach Inzision der Scarpa-Faszie und Dissektion des Subkutangewebes folgt die Darstellung der Externusaponeurose und deren Übergang in das Leistenband nach kaudal. Der Anulus inguinalis superficialis wird aufgesucht (Abb. 5c).

Ausgehend vom Anulus inguinalis superficialis wird nun die Externusaponeurose im Faserverlauf von mediokaudal nach laterokranial gespalten (Abb. 5 d). Dabei empfiehlt sich, die Inzision im oberen Teil des Anulus inguinalis superficialis zu beginnen, um später für die Rekonstruktion ausreichend Material kaudal zu erhalten.

Nach Eröffnung des Leistenkanals liegt der Samenstrang frei. Die kraniale und kaudale Lefze der Externusaponeurose werden mobilisiert, der dem M. cremaster aufliegende $\mathbf{N}$. ilioinguinalis dargestellt und in Abhängigkeit vom anatomischen Verlauf ggf. mobilisiert und wenn möglich unter den kranialen oder kaudalen Haken verlagert und geschont. Bei einer weiten Mobilisation der Externusaponeurose von der Internusmuskulatur nach kranial ist auch der hier oftmals die Externusaponeurose durchbrechende R. cutaneus des $\mathbf{N}$. iliohypogastricus darzustellen und zu schonen.
Im Bereich des Os pubis kann jetzt der Samenstrang vorsichtig stumpf unterfahren und sicher mittels Gummizügel angeschlungen werden. Durch weiteres stumpfes Abschieben des Funiculus vom Leistenband lassen sich nun die Vasa cremasterica externa als sogenannte Blue Line darstellen. Hierbei handelt es sich um kleine Gefäßverbindungen zu den epigastrischen Gefäßen. Je nach anatomischem Verlauf können diese sich am inneren Leistenring aufspannen und dadurch eine suffiziente Einengung des Anulus inguinalis profundus behindern bzw. die Netzplatzierung für die Lichtenstein-Reparation erschweren. In diesem Fall sollten diese Gefäße zwischen Klemmen durchtrennt und ligiert werden. Dabei ist unbedingt auf den R. genitalis zu achten, der häufig in enger Nachbarschaft zu diesen Gefäßen verläuft und nicht in die Gefäßligatur mit einbezogen werden sollte. Sollte der R. genitalis nicht erhalten werden können, wird

\section{Hintergrund}

\section{Aktuelle Studien}

Generell wird das Handling der Nerven intraoperativ und der Einfluss auf die Ausbildung eines chronischen Schmerzsyndroms kontrovers diskutiert. Eine routinemäßige, prophylaktische Neurektomie des N. ilioinguinalis während der Intervention wird aufgrund zweier aktueller Metaanalysen nicht empfohlen. Dagegen könnte die Identifikation aller relevanten Nerven das Risiko eines intraoperativen Nervenschadens und die Ausbildung eines Schmerzsyndroms reduzieren (Simons et al. 2009). Ein systematischer Review konnte weiterhin einen signifikanten Effekt zur Vorbeugung eines postoperativen Schmerzsyndroms durch die bei der Lokalanästhesie per se durchgeführte Blockade aller relevanten Nerven nachweisen (Joshi et al. 2012), sodass dieses Vorgehen bei allen Patienten im Rahmen der offenen Reparation einer Leistenhernie von den Leitlinien der Europäischen Herniengesellschaft empfohlen wird (Miserez et al. 2014). 

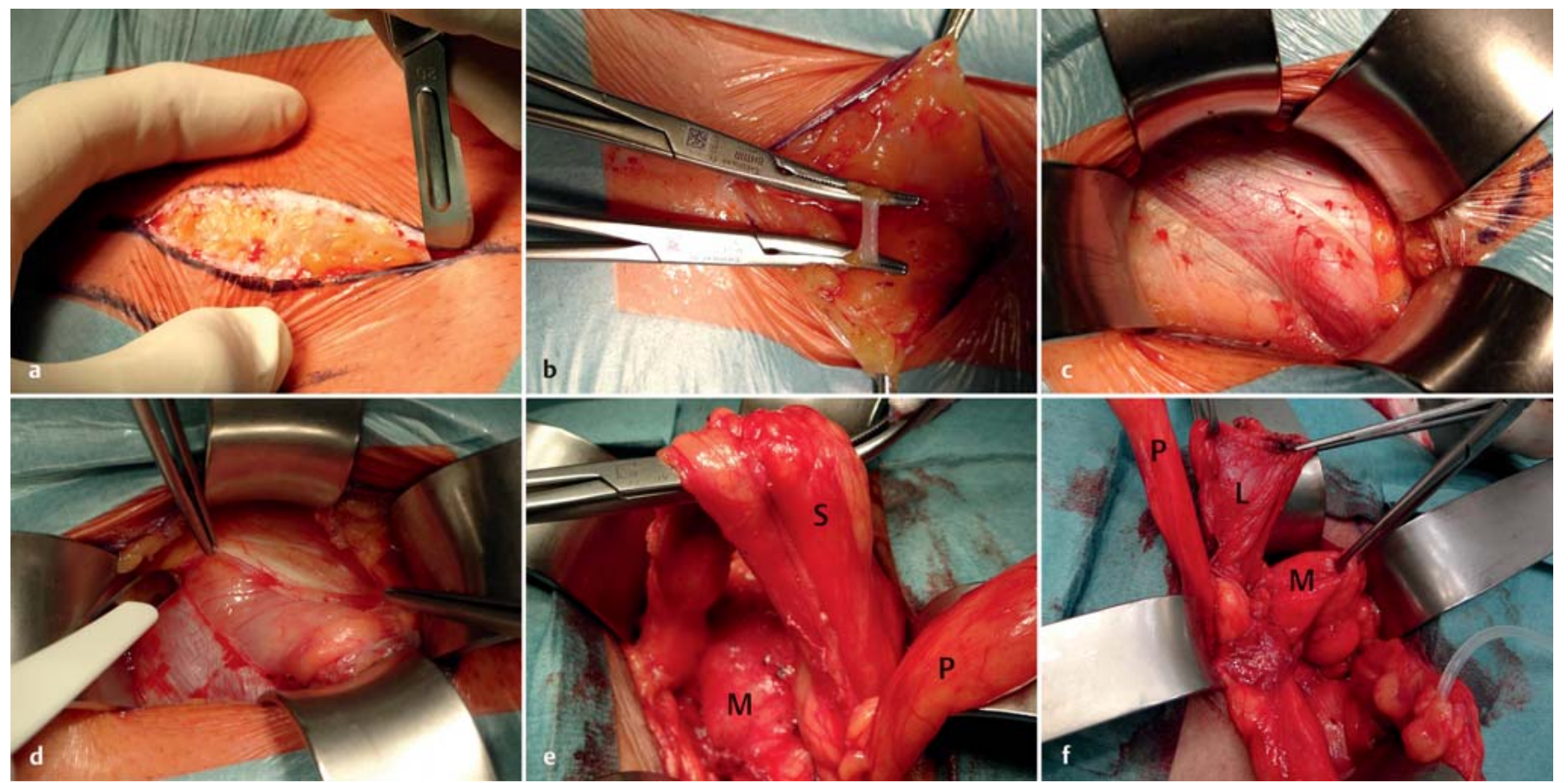

Abb. 5 - Operatives Vorgehen. OP-Schritte der Präparation. a Hautinzision als Querschnitt im Bereich oberhalb der Leistenbeugefalte bei einer rechtsseitigen Leistenhernie. b Versorgung der subkutan verlaufenden Vasa epigastrica superficilia. c Blick auf die Externusaponeurose mit Darstellung des Anulus inguinalis superficialis. d Eröffnung des Leistenkanals durch Inzision der Externusaponeurose ausgehend vom Anulus inguinalis superficialis von mediokaudal nach laterokranial im Faserverlauf. e Unterfahren und Anzügeln des Samenstrangs (S) nach Separation eines großen lateralen präperitonealen Lipoms (P). Vorwölbung der Fascia transversalis als Leistenkanalhinterwand unter Ausbildung eines medialen Bruchsacks (M). $\mathbf{f}$ Situs nach Separation eines präperitonealen Lipoms (P), eines lateralen Bruchsacks (L) bis in den Bereich des Anulus inguinalis profundus. Medial zeigt sich additiv ein direkter Bruchsack (M). Der Samenstrang ist unter den kaudalen Haken verlagert.

dieser selektiv freigelegt und durchtrennt. Nun kann die Leistenkanalhinterwand beurteilt bzw. hier vorliegende mediale Fasziendefekte dargestellt werden (Abb. 5e).

Bei lateralen, indirekten Hernien erfolgt die Längsinzision des den Samenstrang umgebenden $\mathbf{M}$. cremaster und ggf. dessen sparsame Resektion. Dabei ist auf den dorsal verlaufenden R. genitalis des N. genitofemoralis zu achten. Es können jetzt vorliegende präperitoneale Lipome auspräpariert, bis zum inneren Leistenring (Anulus inguinalis profundus) dargestellt und hier abgesetzt werden. Der laterale Bruchsack wird schrittweise von den Samenstranggebilden separiert und bis zu seiner peritonealen Umschlagsfalte freigelegt. Auf eine ausreichend zirkuläre Freilegung ist hierbei unbedingt zu achten, um eine spannungsarme Reposition des Bruchsacks zu ermöglichen (Abb. 5f).

Nach vollständiger Präparation und Abtragung eines präperitonealen Lipoms können die Bruchsäcke eröffnet und der Bruchsackinhalt versorgt werden. In der Regel kann jedoch auf eine Eröffnung und Inspektion des Inhalts verzichtet werden. Oftmals reicht, vor allem bei lateralen Bruchsäcken, die Invertierung und Reposition aus. Eine Versorgung mittels Tabaksbeutelnaht oder die Torsion und die tiefe Umstechung und Abtragung des Bruchsacks ist nur in Ausnahmefällen bei großen Befunden (z.B. Skrotalhernien) indiziert.

\section{- Lichtenstein-Reparation}

Siehe hierzu Abb. 6.

Im Rahmen der Originalbeschreibung der LichtensteinReparation wurde der Bruchsack nach entsprechender Präparation ohne Eröffnung reponiert und die Bruchpforten ohne weitere Behandlung mit der Netzprothese lediglich überdeckt. Zur Sicherung des Repositionsergebnisses und um ein Netzbulging gerade der bevorzugten großporigen elastischen Netzprothesen zu vermeiden, empfiehlt sich eine fortlaufende Naht zwischen kranialem und kaudalem Rand der Fascia transversalis von medial nach lateral (Abb. 6b). Diese Naht kann mit resorbierbarem Nahtmaterial (z. B. Vicryl 2-0) erfolgen.

Die eingebrachte Netzprothese (Abb.6c) wird medial über dem Os pubis mit einer Überlappung von mindestens $1 \mathrm{~cm}$ durch eine $U$-Naht aus nicht resorbierbarem Material (z.B. Prolene 2-0) fixiert, ohne jedoch das sensible Periost mitzufassen. Diese Naht wird anschließend 


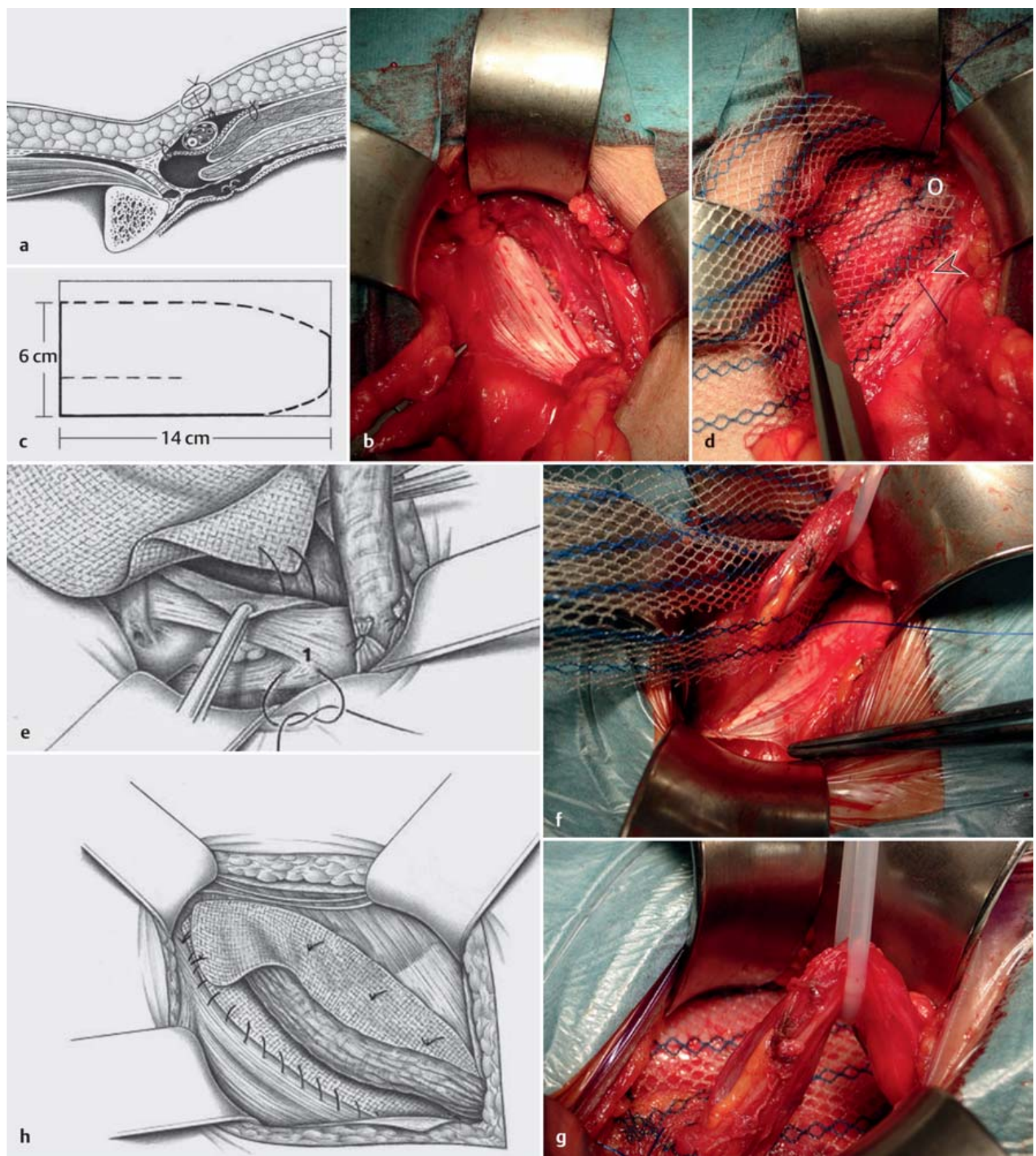

Abb. 6 - OP-Schritte der Lichtenstein-Reparation. a Lage des Netzes bei der Lichtenstein-Reparation (aus: Schumpelick M. Hernien. 4. Aufl. Stuttgart: Thieme; 2000). b Raffung der Leistenkanalhinterwand durch fortlaufend adaptierende Naht. c Größe und Zuschnitt der Netzprothese (aus: Schumpelick M. Hernien. 4. Aufl. Stuttgart: Thieme; 2000). d Platzierung der Netzprothese und Fixation über dem Os pubis (O) mit einer Überlappung von $1 \mathrm{~cm}$ und anschließender Fixation der fortlaufenden Naht des kaudalen Netzrands an die Basis des Leistenbands. e Mediale Netzfixation im Bereich des Lig. Cooperi (1) bei vorliegender Femoralhernie (aus: Schumpelick M. Hernien. 4. Aufl. Stuttgart: Thieme; 2000). f Führung der jetzt von lateral eingeschnittenen Netzprothese um den inneren Leistenring. $\mathbf{g}$ Rekonstruktion des inneren Leistenrings durch Fixation des kaudalen Endes der kranialen Netzlefze zusammen mit der kaudalen Lefze an die Basis des Leistenbands. h Schemazeichnung des vollständig platzierten und fixierten Netzes. (Aus: Schumpelick M. Hernien. 4. Aufl. Stuttgart: Thieme; 2000.) 
fortlaufend unter Anheftung des kaudalen Netzrands an die Basis des Leistenbands bis lateral des inneren Leistenrings fortgeführt (Abb. $\mathbf{6 d}$ ). Aufgrund der anatomisch variablen Länge der Leistenkanalhinterwand empfiehlt es sich aus eigener Erfahrung, erst jetzt die Netzprothese von lateral her einzuschneiden. Dies erfolgt $2 \mathrm{~cm}$ kranial des kaudalen Netzrands soweit nach medial, dass ohne Einschnürung des Samenstrangs die kraniale Lefze des Netzes um diesen herumgeführt werden kann. Bei Verwendung von vorgefertigten, geschlitzten LichtensteinNetzen sollte die Distanz zwischen Os pubis und innerem Leistenring entsprechend vor Netzplatzierung ausgemessen und die Netzprothese ggf. am medialen Rand getrimmt werden.

Sollte sich intraoperativ zusätzlich eine Femoralhernie zeigen, so muss für die Versorgung die kaudale Netzfixierung medial der Femoralgefäße nicht am Leistenband, sondern tiefer am Lig. Cooperi erfolgen, um auch hier eine ausreichende Verstärkung zu erhalten (Abb.6e).

Die medialen und kranialen Netzpartien werden auf dem M. obliquus internus platziert, wobei hier sorgsam auf den aus dem M. obliquus internus tretenden und häufig die Externusaponeurose penetrierenden N. iliohypogastricus zu achten ist. Dabei sollte insbesondere ein direkter Kontakt zwischen Netz und Nerv vermieden werden. Jetzt erfolgt die Rekonstruktion des Anulus inguinalis profundus durch Nahtfixation des kaudalen Randes der kranialen Netzlefze lateral des inneren Leistenrings zusammen mit der darunterliegenden kaudalen Netzlefze (Abb. 6f, g). Die lateral überstehenden Netzanteile können weiter nach lateral unter die Externusaponeurose platziert werden und dort ohne Fixation zwischen Externusaponeurose und M. obliquus internus verbleiben. Medial und kranial erfolgt die punktförmige Fixation des Netzes mit 2-3 Einzelknopfnähten auf der Faszie des M. obliquus internus. Diese Nähte sind vorzugsweise locker mit einem Luftknoten zu knüpfen, um den hier intramuskulär verlaufenden N. iliohypogastricus nicht zu irritieren.

Prinzipien

\section{Lichtenstein-Reparation}

Das Prinzip der Lichtenstein-Reparation ist die Verstärkung der Leistenkanalhinterwand durch ein $14 \times 6 \mathrm{~cm}$ großes Netz, welches hinter dem Samenstrang von der Basis des Leistenbands bis auf die Internusmuskulatur platziert wird und somit zu einer Verstärkung der Leistenkanalhinterwand führt (Abb. 6a).
- Viele Studien haben sich mit der atraumatischen Netzfixation gerade im Hinblick auf die postoperativen Schmerzen bei der Lichtenstein-Reparation beschäftigt. Verglichen wurden nicht resorbierbares sowie resorbierbares Nahtmaterial, Acrylatkleber, Fibrinkleber und selbstfixierende Netze. Außer z.T. kurzfristigen postoperativen Effekten konnte jedoch für die atraumatische Netzfixation kein Langzeiteffekt auf die Rate chronischer Schmerzen nachgewiesen werden. Diese Ergebnisse rechtfertigen aus unserer Sicht aktuell nicht den routinemäßigen Einsatz, gerade im Hinblick auf die zumeist deutlich höheren Kosten der atraumatischen Netzfixation (Abb. 6h).

Es folgt der von lateral beginnende Verschluss der Externusaponeurose über dem Netz und abschließend die Naht von Scarpa-Faszie und der Hautverschluss. Auf eine Redon-Drainage kann üblicherweise verzichtet werden.

\section{- Shouldice-Reparation}

Siehe hierzu Abb. 7.

Die allgemeinen Präparationsschritte und die Versorgung der Bruchpforten erfolgen analog zum obig beschriebenen Vorgehen.

Es folgt die Darstellung und Längsinzision der Fascia transversalis vom Anulus inguinalis profundus bis zum Os pubis unter sorgfältiger Schonung der präperitoneal verlaufenden epigastrischen Gefäße. Die kraniale Lefze der Fascia transversalis (Abb. 7 b) wird retroperitoneal nach kranial mobilisiert und somit der laterale Rand der Rektusscheide, die sog. White Line dargestellt (Abb. 7c). Die ausreichende Darstellung der White Line sichert auch bei anderweitig sehr ausgedünnter Fascia transversalis ein ausreichendes Nahtlager im kranialen Bereich. Die

\section{Prinzipien}

\section{Verfahren zur Reparation von Leistenhernien}

Das von Edward Earle Shouldice und seinen Mitarbeitern zwischen 1945 und 1953 entwickelte Verfahren zur Reparation von Leistenhernien beruht auf einer Verstärkung der Leistenkanalhinterwand und Einengung des inneren Leistenrings durch Dopplung der Fascia transversalis und Fixation der Transversus- und Internusmuskulatur an die Basis des Leistenbands als relevante Barriere für das Auftreten einer Hernie (Abb. 7a). 


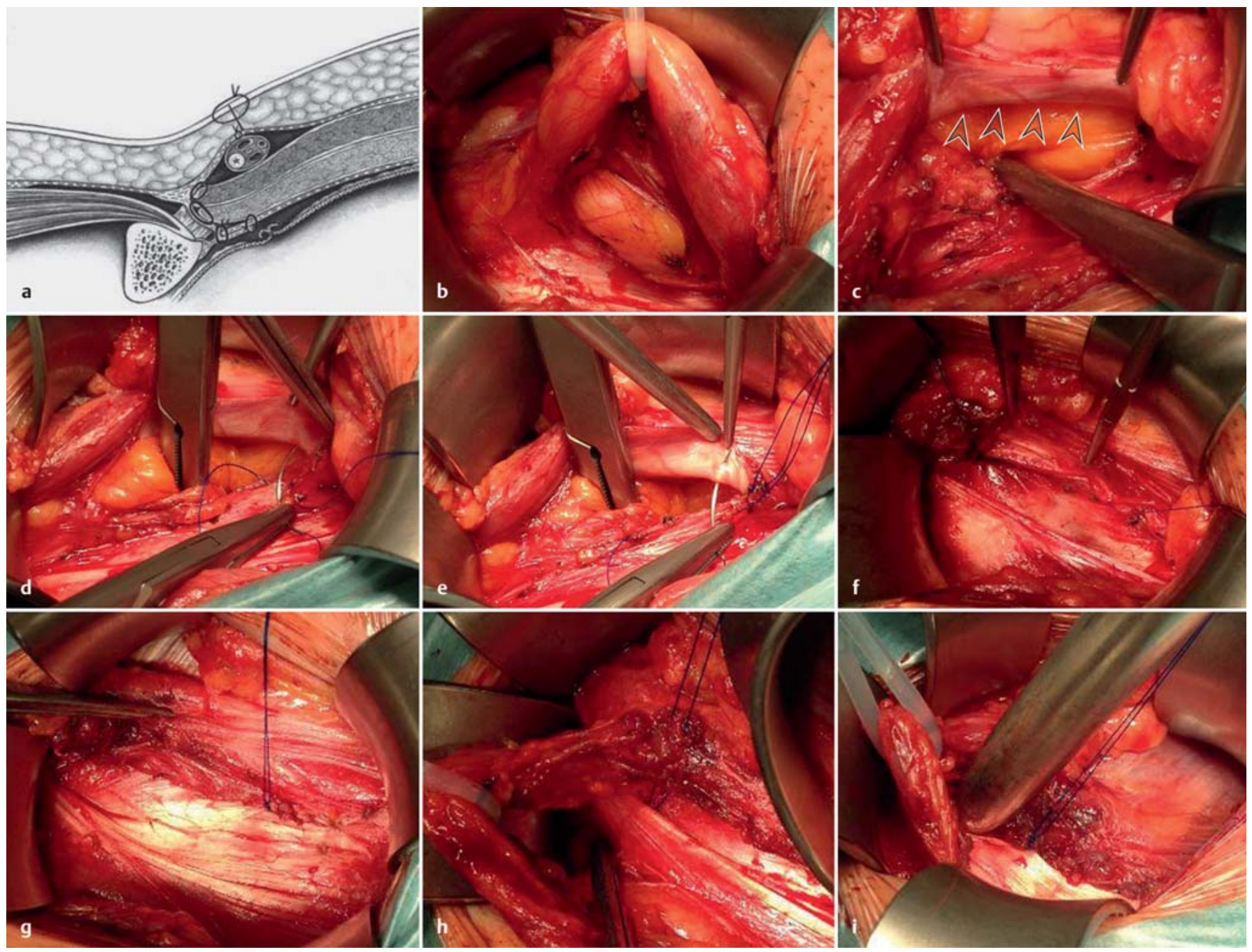

Abb. 7 - OP-Schritte der Shouldice-Reparation. a Schemazeichnung der Shouldice-Reparation mit gedoppelter Fascia transversalis und Fixation der Muskulatur an die Basis des Leistenbands (aus: Schumpelick M. Hernien. 4. Aufl. Stuttgart: Thieme; 2000). b Rechtsseitige Leistenhernie. Angezügelter Samenstrang und Darstellung einer mittleren direkten Hernie mit Vorwölbung der Fascia transversalis (MII). c Darstellung des lateralen Randes der Rektusscheide, die sog. White Line, nach Mobilisation der Fascia transversalis. d Erste Shouldice-Naht medial am Os pubis beginnend unter Fixation der kaudalen Lefze der Fascia transversalis an der White Line. e Weiterführung der ersten Shouldice-Naht. f Erste Nahtreihe der ersten Shouldice-Naht unter Einengung des inneren Leistenrings. g Fertiggestellte erste Shouldice-Naht mit gedoppelter Fascia transversalis. h Beginn der zweiten Shouldice-Naht zur gedoppelten Adaptierung der Transversus- und Internusmuskulatur an das Leistenband. i Überprüfung der ausreichenden Weite des inneren Leistenrings nach abgeschlossener zweiter Shouldice-Naht (z. B. mit Hegar-Stift-Größe 11).

kaudale Lefze wird ebenfalls retroperitoneal bis an den Ansatz zum Leistenband mobilisiert.

Sollte sich hier intraoperativ retroperitoneal eine Femoralhernie zeigen, so kann diese durch Adaptierung des kaudalen Randes des Leistenbands mit dem Lig. Cooperi versorgt werden.

Nach ggf. Resektion ausgedünnter Anteile der Fascia transversalis erfolgt die Anlage der ersten Shouldice-Naht (z.B. Prolene 2-0). Dabei wird medial, direkt am Os pubis beginnend, die kaudale Lefze der Fascia transversalis von unten an der White Line fixiert (Abb. 7d,e). Die Naht er- folgt fortlaufend bis zum inneren Leistenring, welcher auf Zeigefingerkuppengröße eingeengt werden soll (Abb. 7f). Eine weitere Einengung gefährdet die Durchblutung (insbesondere den venösen Abfluss der Samenstranggefäße). Hier erfolgt die Fadenumkehr und Dopplung der Fascia transversalis (Abb. 7g) durch Fixation der kranialen Lefze an der Basis des Leistenbands. Diese Naht sollte bis weit nach medial an das Os pubis geführt werden und, ggf. zum Knüpfen mit dem Initialfaden, auch ein Rückstich erfolgen. Dabei ist besonders auf die nun nicht mehr sichtbaren epigastrischen Gefäße und auch auf die Femoralgefäße zu achten, die bei tiefen Stichen mit erfasst werden können. 
Die zweite Shouldice-Naht erfolgt ebenfalls fortlaufend, lateral am inneren Leistenring beginnend, unter Anheftung der Transversus- und Internusmuskulatur an die Basis des Leistenbands bis nach medial zum Os pubis und unter Umkehr zurück zum inneren Leistenring (Abb. 7 h). Jetzt kann erneut die Weite des inneren Leistenrings kontrolliert werden (Abb. 7i).

Analog zum Vorgehen bei der Lichtenstein-Reparation folgt der von lateral beginnende fortlaufende Verschluss der Externusaponeurose (z. B. Monocryl 3-0) und abschließend die Naht von Scarpa-Faszie und der Hautverschluss.

\section{Weitere Verfahren zur offenen Leistenhernienreparation}

Neben den beschriebenen Verfahren existieren noch zahlreiche weitere Verfahren und Modifikationen zur offenen Reparation einer Leistenhernie. Zu den weiteren Nahtverfahren gehören das Verfahren nach Zimmerman, der Minimal Repair nach Muschaweck oder die Reparation nach Lotheissen/McVay, bei denen jeweils nur lokal die Bruchpforte versorgt wird. Diese Verfahren sind indiziert v.a. bei kleinen Defekten mit ansonsten intakter und belastbarer Fascia transversalis, welche ein ausreichend stabiles Nahtlager ermöglicht. Ein in Europa bislang noch wenig bekanntes Verfahren ist die vom indischen Chirurgen Mohan Desarda 2001 beschriebene Technik, bei der eine netzfreie Verstärkung der Hinterwand mit einem Teil der Externusaponeurose als Faszienverschiebelappenplastik erfolgt (Desarda 2001).

Zu den weiteren Netzverfahren gehört die transinguinale präperitoneale Patchplastik, bei der über einen anterioren Zugang das Netz im Präperitonealraum platziert und fixiert wird. Bei diesem Verfahren entspricht die Netzposition der bei endoskopischen Verfahren (transabdominale präperitoneale Netzplastik, totale extraperitoneale Netzplastik). Ebenfalls präperitoneal werden die Netze bei der Rives-Technik, dem Kugelpatch oder der präperitonealen Reparation nach Pelessier oder Ugahary platziert. Dreidimensionale Netze, ggf. in Kombination mit einem in Lichtenstein-Position eingebrachten flachen Netzanteil, finden beim Plug- bzw. Plug-und-PatchVerfahren bzw. bei der Gilbert-Technik mit dem Prolene Hernia System oder dem Ultrapro Hernia System Anwendung.

\section{Nachsorge}

Generelle Einschränkungen können dem Patienten postoperativ nicht empfohlen werden. Es gilt das Prinzip: „Do what you can feel you can do“. Vermutlich ist sogar die früher gegebene Einschränkung, schweres Heben und stärkere Belastung für einige Wochen zu vermeiden, heute obsolet. Neuere Untersuchungen konnten aufzeigen, dass beim Niesen oder Husten ein deutlich höherer intraabdomineller Druck die Leiste belastet.

\section{Fazit}

In den letzten Jahren zeichnet sich ein deutlicher Verfahrenswechsel bei der Versorgung von Leistenhernien ab. Wurden vor 20 Jahren fast ausschließlich Nahtverfahren eingesetzt, stehen heute vor allem die Netzverfahren im Vordergrund. Dies wird wissenschaftlich unterstützt durch die Leitlinien der Europäischen Herniengesellschaft, welche generell die Implantation eines Netzes bei der Leistenhernienreparation empfehlen. Diese Empfehlungen beruhen auf aktuellen Studien und Metaanalysen, nicht aber auf einer patientenadaptierten Verfahrenswahl, dem sog. Tailoring. Bei den offenen Verfahren kann die „Netzentscheidung“ intraoperativ getroffen werden, in Abhängigkeit von der jeweils vorliegenden Anatomie und Morphologie der Hernie und den patientenspezifischen Risikofaktoren.

Leistenhernienrezidive sollten nach laparoskopischer Reparation eher von anterior mit einem offenem Verfahren versorgt werden. Die Möglichkeit, diesen Eingriff auch in Lokalanästhesie mit oder ohne Analgosedierung durchführen zu können, ist nicht nur für Hernienpatienten mit Narkoserisiko interessant. Die offenen Verfahren in der Leistenhernienchirurgie, ob mit oder ohne Netz, haben weiterhin einen wichtigen Stellenwert und sollten von allen Chirurgen, welche Leistenhernien operieren, beherrscht werden.

Interessenkonflikt: Die Autoren bestätigen, dass kein Interessenkonflikt vorliegt. 


\section{Literatur}

Amato B, Moja L, Panico S et al. Shouldice technique versus other open techniques for inguinal hernia repair. Cochrane Database Syst Rev 2012; 4: CD001543

Chung L, Norrie J, O’Dwyer PJ. Long-term follow-up of patients with a painless inguinal hernia from a randomized clinical trial. Br J Surg 2011; 98: 596 - 599

Desarda MP. New method of inguinal hernia repair: a new solution. ANZ J Surg 2001; 71: $241-244$

Fitzgibbons RJ jr., Giobbie-Hurder A, Gibbs JO et al. Watchful waiting vs repair of inguinal hernia in minimally symptomatic men: a randomized clinical trial. JAMA 2006; 295: 285-292

Fitzgibbons RJ jr., Ramanan B, Arya S et al. Long-term Results of a Randomized Controlled Trial of a Nonoperative Strategy (Watchful Waiting) for Men With Minimally Symptomatic Inguinal Hernias. Ann Surg 2013; 258: $508-515$

Joshi GP, Rawal N, Kehlet $\mathrm{H}$ et al. Evidence-based management of postoperative pain in adults undergoing open inguinal hernia surgery. $\mathrm{Br}$ J Surg 2012; 99: $168-185$

Junge $\mathrm{K}$, Rosch $\mathrm{R}$, Klinge $\mathrm{U}$ et al. Risk factors related to recurrence in inguinal hernia repair - a retrospective analysis. Hernia 2006; 10 : 309-315

Köckerling F, Jacobs D, Grund S et al. Prinzipien der minimalinvasiven Chirurgie bei Hernien Teil 2. Allgemein- und Viszeralchirurgie up2date 2012; 2: 99-115

Miserez M, Alexandre JH, Campanelli G et al. The European hernia society groin hernia classification: simple and easy to remember. Hernia 2007; 11: $113-116$

Miserez M, Peeters E, Aufenacker T et al. Update with level 1 studies of the European Hernia Society guidelines on the treatment of inguinal hernia in adult patients. Hernia 2014; 18: 151-116

O'Dwyer PJ, Norrie J, Alani A et al. Observation or Operation for Patients With an Asymptomatic Inguinal Hernia - A Randomized Clinical Trial. Ann Surg 2006; 244: 167-173

Schumpelick M. Hernien. 4. Aufl. Stuttgart: Thieme; 2000

Simons MP, Aufenacker T, Bay-Nielsen M et al. European Hernia Society guidelines on the treatment of inguinal hernia in adult patients. Hernia 2009; 13: 343-403

\section{Korrespondenzadresse}

Prof. Dr. med. K. Junge

Klinik für Allgemein-, Viszeral- und minimalinvasive Chirurgie Medizinisches Zentrum StädteRegion Aachen $\mathrm{GmbH}$ Mauerfeldchen 25

52146 Würselen

Telefon: 02405-623002

Fax: $\quad 02405-623004$

E-Mail: karsten.junge@mz-ac.de 


\section{Bauchwand, Zwerchfell, Retroperitoneum}

\section{CME-Fragen}

\section{CME•thieme.de}

\section{CME-Teilnahme}

- Viel Erfolg bei Ihrer CME-Teilnahme unter http://cme.thieme.de

- Bitte informieren Sie sich vorab online über die Gültigkeitsdauer.

- Sollten Sie Fragen zur Online-Teilnahme haben, unter http://cme.thieme.de/hilfe finden Sie eine ausführliche Anleitung.

Die Leistenhernienreparation stellt durchschnittlich wie viel Prozent aller chirurgischen Eingriffe dar?
A $<5 \%$
B $10-15 \%$
C $30-50 \%$
D $60-80 \%$
E $>90 \%$

Wie viel Prozent der Männer müssen im Laufe ihres Lebens mit dem Auftreten einer

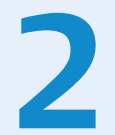

A $5 \%$

B $15 \%$

C $25 \%$

Leistenhernie rechnen?

D $35 \%$

E $45 \%$

Welcher pathophysiologische Aspekt gilt nicht direkt als Risikofaktor für das Auftreten einer Leistenhernie?

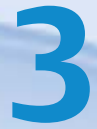

A positive Familienanamnese

B Kollagenerkrankungen

C persistierend offener Processus vaginalis

D Aszites

E chronisch-entzündliche Darmerkrankungen
Welche Struktur bildet einen Teil der Hinterwand des Leistenkanals?
A Fascia transversalis

B M. rectus abdominis

C Aponeurose des M. obliquus externus abdominis

D Leistenband

E Lig. Cooperi

Wo treten direkte Leistenbrüche auf?

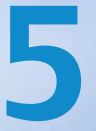

A Lacuna vasorum

B Fossa inguinalis medialis

C Fossa inguinalis lateralis

D Fossa obturatoria

E Fossa ischiorectalis 
Was ist ein typisches Primärsymptom einer Leistenhernie?
A reponible Schwellung
B Hämatochezie
C Diarrhö
D Hämatemesis
E Nykturie

Was gehört nicht zur Routinediagnostik einer unkomplizierten Leistenhernie?

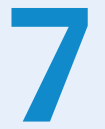
A Anamnese
B klinischer Befund
C Palpation
D Sonografie
E CT/MRT

Welches Operationsverfahren kann nicht in Lokalanästhesie durchgeführt werden?
A Shouldice

B Bassini

C transabdominale präperitoneale Netzplastik

D Lichtenstein

E Plug und Patch
Was ist das Reparationsprinzip des Shouldice-Verfahrens?
A Verstärkung der Leistenkanalhinterwand mit einem Kunststoffnetz

B Verstärkung der Leistenkanalvorderwand mit einem Kunststoffnetz

C Verstärkung der Leistenkanalhinterwand durch Dopplung der Fascia transversalis

D Verstärkung der Leistenkanalvorderwand durch Dopplung der Fascia transversalis

E Raffung der Externusaponeurose zur Verstärkung der Leistenkanalvorderwand
Was ist das Reparationsprinzip des Lichtenstein-Verfahrens?
A Verstärkung der Leistenkanalhinterwand mit einem Kunststoffnetz

B Verstärkung der Leistenkanalvorderwand mit einem Kunststoffnetz

C Verstärkung der Leistenkanalhinterwand durch Dopplung der Fascia transversalis

D Verstärkung der Leistenkanalvorderwand durch Dopplung der Fascia transversalis

E Raffung der Externusaponeurose zur Verstärkung der Leistenkanalvorderwand 